\title{
Modelling of the dynamic behaviour of hard-to-machine alloys
}

\author{
M. Hokka ${ }^{1}$, T. Leemet ${ }^{1}$, A. Shrot ${ }^{2}$, M. Bäker ${ }^{2}$, and V.-T. Kuokkala ${ }^{1}$ \\ ${ }^{1}$ Tampere University of Technology, Department of Materials Science, Finland \\ 2 Technische Universität Braunschweig, Institute for Materials, Germany
}

\begin{abstract}
Machining of titanium alloys and nickel based superalloys can be difficult due to their excellent mechanical properties combining high strength, ductility, and excellent overall high temperature performance. Machining of these alloys can, however, be improved by simulating the processes and by optimizing the machining parameters. The simulations, however, need accurate material models that predict the material behaviour in the range of strains and strain rates that occur in the machining processes. In this work, the behaviour of titanium 15-3-3-3 alloy and nickel based superalloy 625 were characterized in compression, and Johnson-Cook material model parameters were obtained from the results. For the titanium alloy, the adiabatic Johnson-Cook model predicts softening of the material adequately, but the high strain hardening rate of Alloy 625 in the model prevents the localization of strain and no shear bands were formed when using this model. For Alloy 625, the Johnson-Cook model was therefore modified to decrease the strain hardening rate at large strains. The models were used in the simulations of orthogonal cutting of the material. For both materials, the models are able to predict the serrated chip formation, frequently observed in the machining of these alloys. The machining forces also match relatively well, but some differences can be seen in the details of the experimentally obtained and simulated chip shapes.
\end{abstract}

\section{Introduction}

Metal alloys with superior mechanical properties are needed in various parts and components of the aerospace, transportation, mining and excavation, and power generation industries. In these alloys, high strength and hardness are combined with good overall ductility, high chemical and corrosion resistance, and excellent fatigue and high temperature performance. Titanium alloys and nickel based superalloys are examples of metal alloys with these extraordinary properties. However, the good mechanical performance can lead to problems in the manufacturing of parts and components. Especially machining of these materials can be challenging, time consuming, and expensive. Most titanium alloys have rather low thermal conductivity, and therefore the temperature of the deforming material often increases rapidly during high speed cutting. The increased temperature of the machining process leads to rapid tool wear and decreased machining quality. Some alloys also produce continuous chips that do not segment and break during machining leading to dangerous entanglements of sharp chips and forcing to interrupt the machining process to manually remove the chips. Also the chemical affinity of titanium can lead to severe galling of the tool and the material causing severe damage to the tool. The nickel based alloys, on the other hand, can contain extremely hard precipitates, such as carbides or intermetallic compounds, which abrade the tool during machining. For these reasons, many of the titanium and nickel based alloys are considered hard-to-machine, and the majority of the overall costs of geometrically complex components can be due to machining. For a better overview on the machining of titanium and nickel alloys see, for example, refs. [8-10].

Machining of these alloys can be improved by developing alloys that are more suitable for machining and by improving the machining processes and tools. Finite element simulations can be very useful in obtaining information about the machining processes and optimizing the machining parameters, such as cutting depth and speed. The simulations, however, require accurate material models based on real experiments. Mechanical testing, on the other hand, can usually be done only in a relatively narrow range of strain, strain rate, and temperature. In machining, however, the strains and strain rates can be significantly higher than what can be studied in a controlled laboratory environment. Therefore, the material models intended for machining simulations must be able to predict the material behaviour also at much higher strains and strain rates than for what they were initially generated. In this work, the mechanical behaviour of titanium 15-3-3-3 alloy and Alloy 625, similar to Inconel 625, was characterized in compression at a wide range of strain rates at room and elevated temperatures. The Johnson-Cook material model $[6,7]$ parameters were obtained from the results of compression tests, and the models were used to simulate orthogonal cutting of the studied alloys. This paper presents and discusses the results of the mechanical testing and modelling of the material behaviour. Preliminary cutting simulation results are also presented and the results are compared with cutting experiments.

\section{Experimental}

The materials studied in this work were Alloy 625 (similar to Inconel 625) and the metastable beta titanium 15-3-33 alloy. Both materials were studied in a fully annealed state without precipitation treatments. The mechanical behaviour of the alloys was characterized in compression in a wide range of strain rates and temperatures. The low strain rate tests were performed with an MTS servohydraulic materials testing machine with an induction heating system for high temperatures. The high strain rate tests were done using a Hopkinson Split Bar device with high temperature capabilities. The high temperature system used in this 

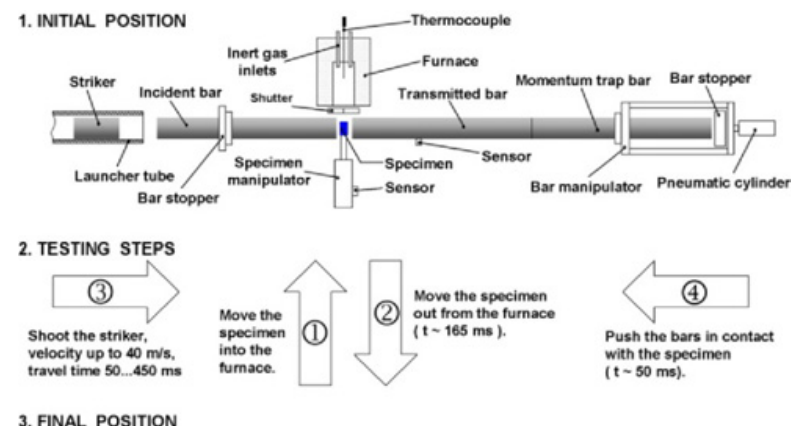

3. FINAL POSITION

Fig. 1. Testing steps in a high temperature Hopkinson Split Bar test.

study heats the specimen in a furnace located beside the bars and utilizes two separate pneumatic manipulation systems for the specimen and the bars. The small cylindrical specimen with a diameter of $6 \mathrm{~mm}$ and length between 6 and $8 \mathrm{~mm}$ is placed in a soft ceramic wool ring. The ring and the specimen are inserted in a special specimen holder arm, which can be pneumatically pushed from the centreline of the bars to the furnace, where it is heated to the test temperature. After heating the specimen holder quickly retracts the specimen back to the centreline of the bars. The striker bar is shot and the second pneumatic actuator pushes the cold bars into contact with the hot specimen just a fraction of a second before the impact loading. The current specimen and bar manipulation system limits the contact time between the hot specimen and the cold bars to less than $50 \mathrm{~ms}$, which guarantees that only the surface temperature of the specimen drops by a few degrees [1-3]. Figure 1 shows the testing steps involved in a high temperature test.

\section{Results}

Figure 2 shows the stress-strain curves at different strain rates and temperatures for the titanium 15-3-3-3 alloy. At room temperature, the strain hardening rate observed in the low strain rate region is significantly higher than that observed at higher strain rates. At the strain rate of $3300 \mathrm{~s}^{-1}$, the strain hardening rate is essentially zero after the initial pronounced yielding. This behaviour can at least partly be explained with adiabatic heating and consequent thermal softening at high strain rates. At higher temperatures, the strength of the material clearly decreases. The strain hardening rate is also much higher at elevated temperatures compared to that at room temperature. Also the strain rate sensitivity of the material was found to increase significantly with temperature.

Figure 3 shows the stress-strain curves obtained at different strain rates and temperatures for Alloy 625. The behaviour of Alloy 625 is significantly different from that observed for the titanium alloy. The stress strain curves at all strain rates and temperatures are nearly linear. At room temperature, the strain hardening rate of the material is essentially constant with respect to strain rate, which also leads to constant strain rate sensitivity with respect to

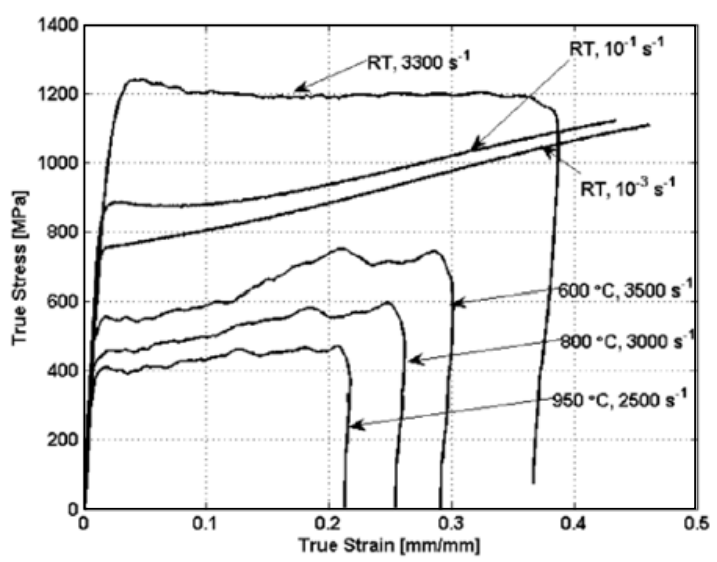

Fig. 2. Results of the compression tests for titanium 15-3-3-3 alloy.

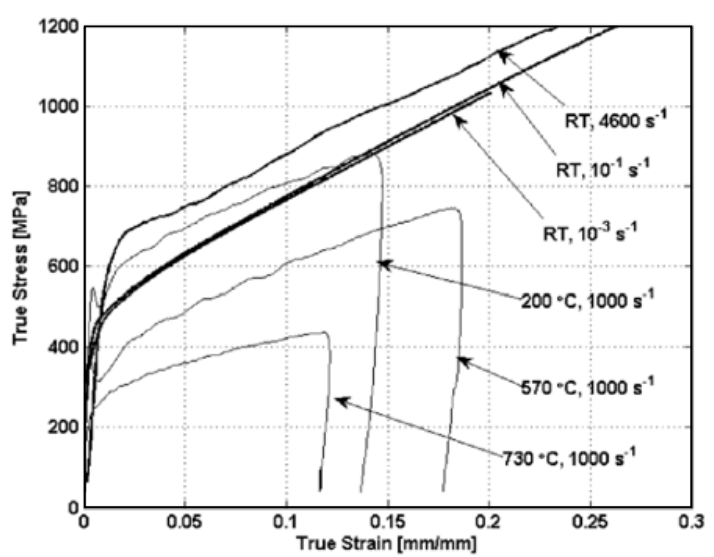

Fig. 3. Results of the compression tests for Alloy 625 .

plastic strain. The strain hardening rate decreases slowly as the temperature is increased obviously due to increased recovery and annihilation of dislocations.

Figure 4 shows the strength of the material as a function of temperature at a constant strain rate. The strength of the titanium 15-3-3-3 alloy decreases rapidly when the temperature is increased from room temperature to $400{ }^{\circ} \mathrm{C}$. Increasing the temperature from $400^{\circ} \mathrm{C}$ to $950^{\circ} \mathrm{C}$ decreases the strength even further, but the stress- strain response is now nearly linear. For Alloy 625, the strength decreases with temperature steadily, but there is more scatter in the results. The temperature sensitivity of the titanium alloy seems to be stronger in the low temperature region than that observed for Alloy 625, but at higher temperatures the strength of the Alloy 625 actually seems to decrease slightly faster. The relative decrease of strength for the titanium alloy is also higher than that for Alloy 625.

\subsection{Modelling}

Modeling of the mechanical behaviour of the titanium alloy is complicated by its strong strain rate sensitivity of strain hardening. A model describing the behaviour in the whole range of strain rates and temperatures would be quite complex, because it needs to take into account the 


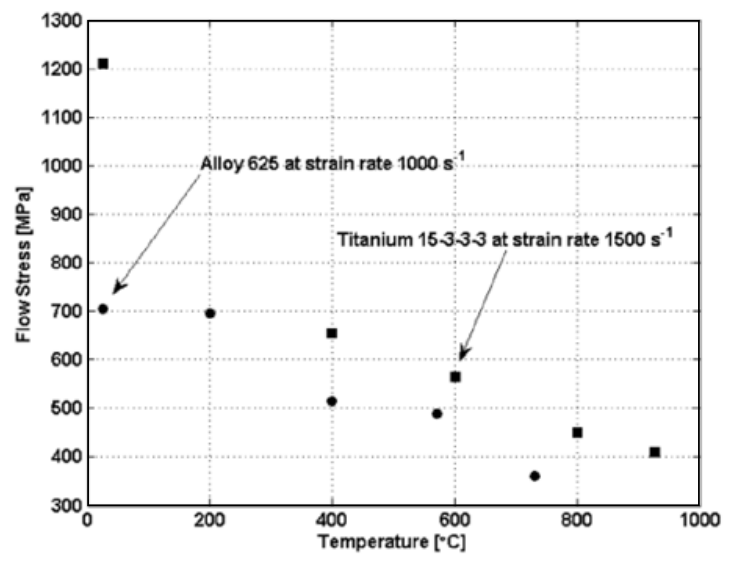

Fig. 4. The temperature dependence of the studied materials at the strain rate of $1000 \mathrm{~s}^{-1}$.

changes in the strain hardening rate with respect to strain rate and temperature. Also, the strain rate sensitivity of the material is not constant, especially at low strain rates, where the sensitivity increases strongly with temperature. Furthermore, especially at room temperature the strain rate sensitivity increases significantly at strain rates around $10^{3} \mathrm{~s}^{-1}$, where the dislocation drag mechanisms become active. The commonly used Johnson - Cook (JC) model can in general be used to model the isothermal response of the material in a narrow range of strain rates. In machining the strain rates can be very high, and therefore the parameters of the JC model were obtained from the compression tests using only the high strain rate data. However, at these high strain rates the material is likely to heat up significantly due to the adiabatic heating. Therefore, the measured adiabatic curves were first converted to isothermal ones using Equation (1). The parameter $m$ used in this Equation was first obtained from the yield strengths of the material measured at the reference strain rate at the reference temperature and at an elevated temperature as shown in Equation (2). However, the obtained parameter $\mathrm{m}$ did not provide good enough results at higher strains and the value of the parameter was manually adjusted to improve the fit of the measured and calculated data also at large strains. The model used to calculate the adiabatic stress is shown in Equation (3).

$$
\begin{gathered}
\sigma_{i}=\frac{\sigma_{a}}{\left[1-\left(\frac{T+\left(\frac{\beta}{\rho c} \int_{0}^{\varepsilon} \sigma d \varepsilon\right)-T_{r}}{T_{m}-T_{r}}\right)^{m}\right]} \\
m=\log \left(1-\frac{\sigma_{Y}^{2}}{\sigma_{Y}^{T r}}\right)-\log \left(\frac{T_{2}-T_{r}}{T_{m}-T_{r}}\right) \\
\sigma_{a}=\left(A+B \varepsilon^{n}\right)\left(1+C_{J C} \ln \frac{\dot{\varepsilon}}{\dot{\varepsilon}_{r e f}}\right) \\
\times\left(1-\left[\frac{T+\left(\frac{\beta}{\rho c} \int_{0}^{\varepsilon} \sigma d \varepsilon\right)-T_{r e f}}{T_{m}-T_{r e f}}\right]^{m}\right)
\end{gathered}
$$

The JC model was used to simulate also the behaviour of Alloy 625. The model parameters were found using a nonlinear fitting routine in Matlab and assuming the
Table 1. Johnson - Cook model parameters for the studied materials.

\begin{tabular}{|l|c|c|}
\hline Parameter & Titanium 15-3 & Alloy 625 \\
\hline $\mathrm{A}$ & 984 & 559 \\
\hline $\mathrm{B}$ & 380 & 2201 \\
\hline $\mathrm{n}$ & 0.3 & 0.8 \\
\hline $\mathrm{C}$ & 0.069 & 0.00021 \\
\hline $\mathrm{m}$ & 1.1 & 1.146 \\
\hline $\mathrm{k}$ & - & 3.00 \\
\hline $\mathrm{T}_{\mathrm{m}}$ & $1669^{\circ} \mathrm{C}$ & $1350^{\circ} \mathrm{C}$ \\
\hline$\dot{\varepsilon}_{\text {ref }}$ & $1400 \mathrm{~s}^{-1}$ & $1670 \mathrm{~s}^{-1}$ \\
\hline
\end{tabular}

deformation to be fully adiabatic. Only the high strain rate data was used since low strain rates hardly occur in machining. However, the strain hardening rate of Alloy 625 is very high at high strain rates, and when extrapolating the material behaviour to larger strains, the model predicts unreasonably high strength values. In the simulations, no serrated chips were formed using this model. This is because the strain does not localize due to the strong strain hardening that effectively compensates for the thermal softening caused by the adiabatic heating. Therefore, the JC model was modified to include also softening at higher strains. An approach similar to that described by Sima and Özel [5] was used in this work by adding an extra strain softening term to the Johnson-Cook model. The modified JC model is shown in Equation (4). The parameters of the Johnson-Cook model for both materials are shown in Table 1.

$$
\sigma_{a}=E q(3) * \tanh \left(\frac{1}{\varepsilon^{k}}\right)
$$

The major differences in the obtained parameters for the two materials are the significantly higher yield strength of the titanium alloy and the higher strain hardening rate of Alloy 625. The strain rate sensitivity of both materials is very low in the high strain rate region.

The high strength and low heat conductivity of the titanium alloy lead to higher adiabatic heating compared to that of Alloy 625. This strong adiabatic heating and consequent thermal softening combined with the relatively low strain hardening rate (parameters $B$ and $n$ ) at high strain rates allow using the Johnson-Cook model for extrapolating the material behaviour to large strains without adding an extra strain softening term to the function. For Alloy 625 , the observed strong strain hardening rate at room temperature, on the other hand, makes the extrapolation impossible without the strength becoming unreasonably high at large deformations.

Figure 5 shows the stress - strain curve obtained at the strain rate of $3300 \mathrm{~s}^{-1}$ for the titanium 15-3-3-3 alloy. The yield behaviour of the material at high strain rates is somewhat pronounced, and the strength decreases after yielding from about $1250 \mathrm{MPa}$ to about $1200 \mathrm{MPa}$. This pronounced yielding was observed at high strain rates, but it was omitted when determining the model parameters. Modelling of the pronounced yielding is somewhat difficult with such a simple equation as the JC model. In addition, the effect of the higher strength at yielding 


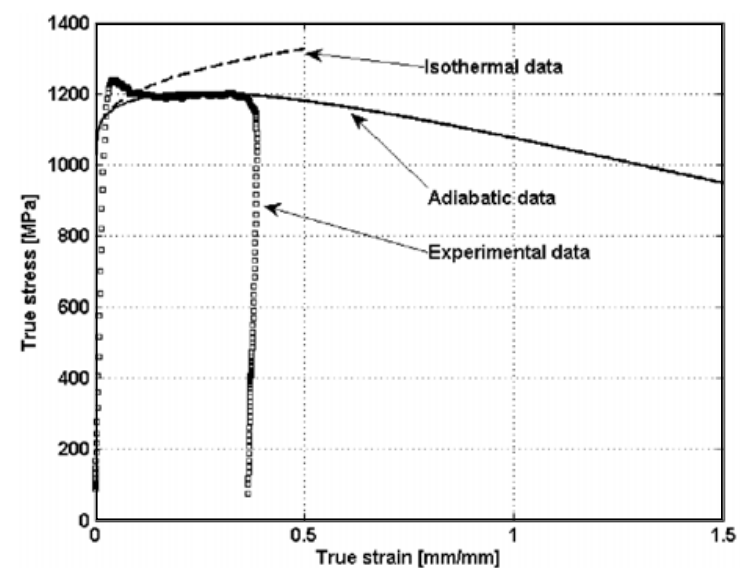

Fig. 5. Experimental data and the isothermal and adiabatic Johnson-Cook model data calculated at strain rate $3300 \mathrm{~s}^{-1}$ for the titanium 15-3-3-3 alloy.

on the chip formation is negligible and can therefore be omitted in the model. The adiabatic data calculated using Equation (3) fits well to the experimentally obtained adiabatic data at the strain rate of $3300 \mathrm{~s}^{-1}$. At higher strains, the adiabatic heating decreases the strength significantly allowing modelling of the strain localization at high strains and strain rates.

Modelling of the behaviour of Alloy 625 requires using the strain softening term in the JC model. The effects of strain softening are shown in Figure 6. Without strain softening, the parabolic hardening function of the model increases the strength of the material above $2000 \mathrm{MPa}$ before the thermal softening becomes a dominant factor and the adiabatic flow stress starts to decrease. This behaviour was found unsuitable for producing segmented chips and shear bands in the tentative simulations of orthogonal cutting. However, using the strain softening exponent $\mathrm{k}=1$ decreases the maximum stress to about $1650 \mathrm{MPa}$. Increasing the strain softening exponent to $\mathrm{k}=2.0$ and $\mathrm{k}=3.0$ actually increases the maximum stress, but the softening following the maximum stress is much stronger than that observed for smaller values of $\mathrm{k}$. For $\mathrm{k}=3.00$, the strength of the material decreases to about $350 \mathrm{MPa}$ at the strain of 2 . The current strain softening term does not essentially affect the simulated flow stresses at small strains, and the match between the experimental data and the simulated values is essentially the same with or without the strain softening term.

\section{Simulations}

ABAQUS 6.9-1 software was used to create a two dimensional explicit finite element model. No heat conduction was allowed between the tool and the chip.

Very fine meshing was used for the workpiece, and the models contained 101151 nodes and 100049 elements, as shown in Figure 7. In the simulations, a conservative friction coefficient of 0.1 was used between the tool and the chip. The separation of the chip from the workpiece was modelled using a sacrificial layer whose, elements were deleted when the shear strain exceeded a predetermined

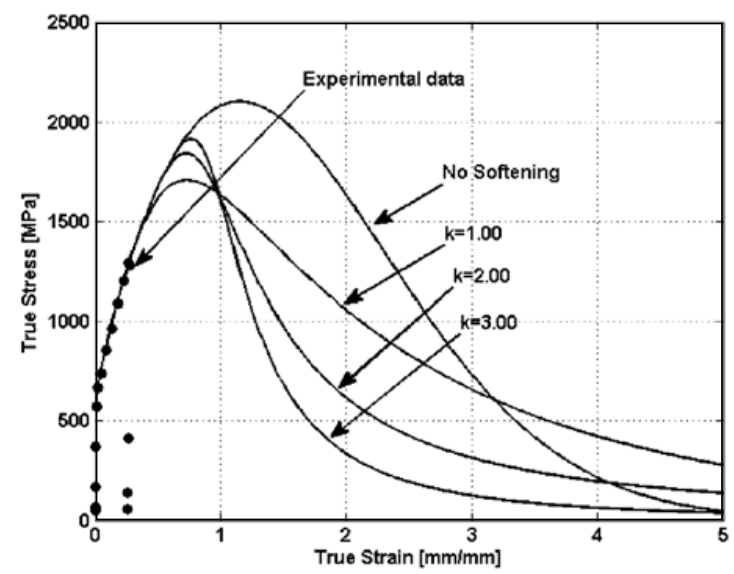

Fig. 6. Experimental data obtained at room temperature at the strain rate of $3500 \mathrm{~s}^{-1}$ and the simulated stress-strain curves with different values of the strain softening exponent $\mathrm{k}$.

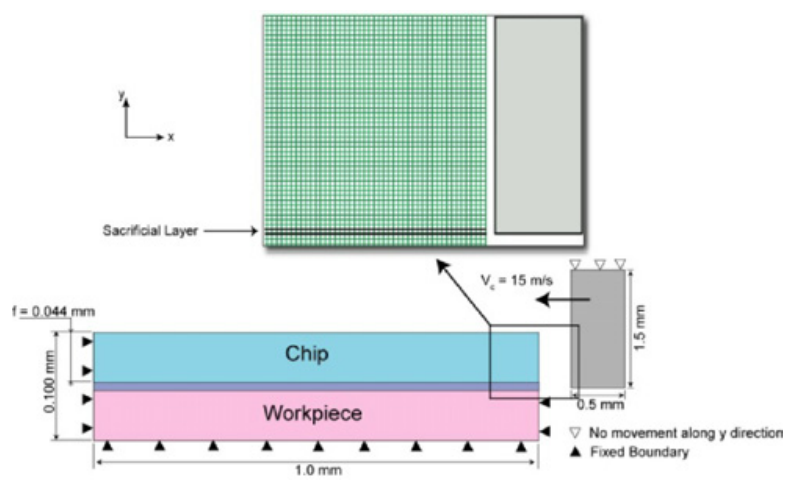

Fig. 7. The finite element model for simulating the orthogonal cutting of the titanium 15-3-3-3 alloy.

value. However, the current material model for Alloy 625 was found unsuitable for the simulations of the separation layer. The extremely low strain rate sensitivity observed at the strain rates near the reference strain rate and the lowered strain hardening rate at high strains lead to rapid softening of the layer at higher strain rates and large strains. The softening of the separation layer leads to a catastrophic crack propagation along the separation layer. Therefore, the strain rate sensitivity of the material model for Alloy 625 was assumed to increase with strain rate, and the parameter ' $C$ ' was calculated using

$$
C(\dot{\varepsilon})=C(1+250) *\left(1-\exp \left(-\left(\dot{\varepsilon}-\dot{\varepsilon}_{0} / T_{c}\right)\right)\right)
$$

where $\mathrm{C}$ is the strain rate sensitivity parameter of the unmodified model, $\dot{\varepsilon}$ the strain rate, $\dot{\varepsilon}_{0}$ is the reference strain rate of the model, and $\mathrm{T}_{\mathrm{c}}$ is a constant with a value of $10^{5} \mathrm{~s}^{-1}$. Also, the $C(\dot{\varepsilon})=\mathrm{C}$ for strain rates lower than the reference strain rate. The value of the strain rate hardening term ranges from 1.3 to 1.45 in the strain rate range of $10^{6}$ to $10^{7} \mathrm{~s}^{-1}$. Therefore there is no discontinuity in the yield stress even after changing $C(\dot{\varepsilon})$ in this way. In the simulations, $\mathrm{C}=C(\dot{\varepsilon})$ was used in both the separation layer and the chip. However, in the separation layer this was done for all strains but in the chip, $\mathrm{C}=C(\dot{\varepsilon})$ was used only for strains higher than 1.5. Ideally, the material model should be the same in both the separation layer and the 


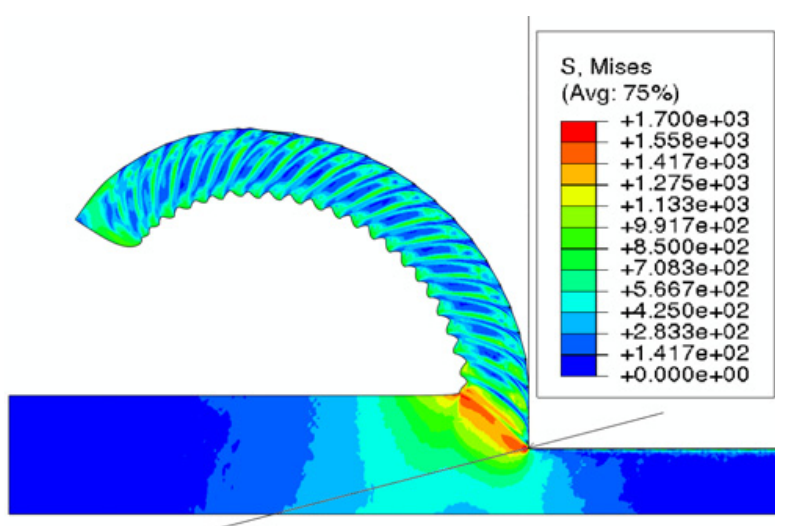

Fig. 8. Simulation results for the titanium 15-3-3-3 alloy.

chip. However, allowing increasing strain rate sensitivity in the chip at low strains will suppress shear localizations and affect the chip shape. This problem needs further attention and will be taken into account in the future work on finite element modelling of Alloy 625.

The results of the simulations were compared to the cutting tests done with the Hopkinson Split Bar device using U-shape specimens. The U-shape test is carried out by inserting the cutting tool between the 'arms' of the $\mathrm{U}$ and placing the specimen-tool assembly between the incident and transmitted stress bars. The test is carried out by impacting the striker to the free end of the incident bar and measuring the cutting forces from the transmitted bar.

The simulations were run using the same cutting speed and depth as in the experiments. The simulation results for the titanium alloy and Alloy 625 are shown in Figures 8 and 9, respectively. For the titanium alloy, serrations are clearly visible, but the chip curvature and overall shape is somewhat different from that observed in machining. For Alloy 625, the serrations are also clear and distinct but much coarser than those observed for the titanium alloy. Closer inspection of the shear bands in Alloy 625 shows splitting of the shear bands near the tool edge, which is also observed in experimentally obtained chips. For both materials, however, the overall shape of the chip as well as the fine details, such as the thickness of individual serrations, are somewhat different from those observed in the experiments.

For the titanium alloy, the cutting forces obtained from the cutting experiments with the U-shape specimen compare quite well. At the cutting speed of $15 \mathrm{~m} / \mathrm{s}$ and cutting depth of $44 \mu \mathrm{m}$, the specific cutting force was $2200 \mathrm{MPa}$, while the simulated cutting force was $2000 \mathrm{MPa}$. The $10 \%$ difference in the cutting forces can at least partly be explained by the different frictional conditions in the experiments and simulations.

For the Alloy 625, the cutting stresses at cutting depth of $47 \mu \mathrm{m}$ and cutting speed of $13 \mathrm{~m} / \mathrm{s}$ obtained from the simulations are significantly higher than the experimentally obtained values. The experimental cutting stress was $1620 \mathrm{MPa}$, whereas the simulated force was $1930 \mathrm{MPa}$. The overestimated cutting force is most likely due to the overestimated strength of the material at high strains.

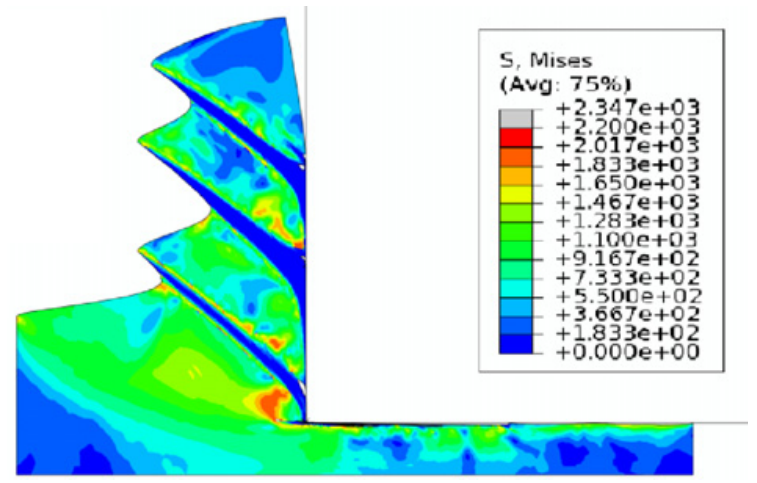

Fig. 9. Simulation results for Alloy 625 .

\section{Summary}

Mechanical behaviour of titanium 15-3-3-3 alloy and a nickel based superalloy, similar to Inconel 625, were studied in this work. The dynamic response of the materials was studied in compression at different strain rates and temperatures. At high strain rates the titanium 15-3-3-3 alloy shows high strength and low strain hardening rate at room temperature. The strength of the material decreases strongly, when temperature is increased from room temperature to $400{ }^{\circ} \mathrm{C}$. At even higher temperatures the strength decreases almost linearly with temperature. Alloy 625 , on the other hand, shows strong and essentially strain rate independent strain hardening at room temperature.

Numerical material models, based on the common Johnson-Cook model, were developed for the studied materials and used in the simulations of orthogonal cutting experiments. Johnson-Cook material model parameters were obtained from the isothermal stress strain curves derived from the initial compression test data at different strain rates and temperatures. For the titanium alloy, the Johnson-Cook model predicts a decrease in strength with increasing strain and adiabatic heating, but for Alloy 625 the model overestimates the strength at large deformations due to its strong parabolic strain hardening term. The model for Alloy 625 was therefore modified to include also the observed strain softening at higher strains.

The simulation results for the titanium alloy show a good match with the experimentally obtained cutting stresses. However, the chip shape and the fine details do not match perfectly and more work is needed to develop the model further. For Alloy 625, the material model predicts strain localisation and splitting of the shear bands near the edge of the tool. However, despite the added strain softening term, the strength predicted by the model is still too high and the simulated cutting stresses clearly higher than those obtained from the cutting experiments.

\section{Acknowledgements}

The research leading to these results has received funding from the European Union Seventh Framework Programme (FP7/20072013) under grant agreement No. PITN-GA-2008-211536, project MaMiNa. 


\section{References}

1. M. Apostol, Ph.D. thesis, Tampere University of Technology (2007)

2. M. Apostol, V.-T. Kuokkala, T. Vuoristo, In: C. Papalettere (Eds.) Advances in Experimental Mechanics (McGraw-Hill, Bari Italy, 2004)

3. M. Apostol, T. Vuoristo, V.-T. Kuokkala, J. de Phys. IV, 110 (2003)

4. D. Gorman, J. Phys. E, 16 (1983).
5. M. Sima, T. Özel, Int. J. Mach. Tools \& Manufacture, $50(2010)$

6. G. Johnson, W. Cook. Int. J. Eng. Fract. Mech. (1985)

7. G. Johnson, W. Cook, Proc. $7^{\text {th }}$ International Symposium on Ballistics (1983)

8. E. O. Ezugwu, Z.M. Wang, A.R. Machado, J. Mat. Proc. Tech., 86,(1999).

9. E.O. Ezugwu, J. Bonney, Y. Yamane,. J. Mat.Proc. Tech. 134, (2003)

10. M. Rahman, Z. Wang, Y. Wong, JSME Int. J., Series C, 49, (2006) 